\title{
APLICAÇÃO DE ESPECTROSCOPIAS RAMAN E INFRAVERMELHO NA IDENTIFICAÇÃO E QUANTIFICAÇÃO DE PLASTIFICANTES EM FILMES COMERCIAIS DE PVC ESTICÁVEL
}

\author{
Michele Lemos de Souza, Paola Corio e Marcia L. A. Temperini* \\ Departamento de Química Fundamental, Instituto de Química, Universidade de São Paulo, CP 26077, 05513-970 São Paulo - SP, \\ Brasil \\ José Aparício Temperini \\ Quimilux C. R. T. Ltda EPP, Rua Gonçalo Pedrosa, 39, 04261-060 São Paulo - SP, Brasil
}

Recebido em 1/7/08; aceito em 19/2/09; publicado na web em 3/7/09

\begin{abstract}
APPLICATION OF INFRARED AND RAMAN TECHNIQUES IN THE IDENTIFICATION AND QUANTIFICATION OF PLASTICIZERS IN COMMERCIAL FILMS OF PVC. This paper reports the use of Raman and infrared techniques for the qualitative and quantitative analysis of plasticizers in polyvinylchloride (PVC) commercial films. FT-Raman marker bands were indentified for di-2-ethyl-hexyl adipate (DEHA) and di-2-ethyl-hexyl phthalate (DEHP), allowing for the rapid identification of these species in the commercial film. Quantitative analysis by FT-IR resulted in plasticizers concentrations ranging from 11 to $27 \%$ (w/w). Considering the little sample preparation and the low cost of the techniques, FT-IR and FT-Raman are viable techniques for a first assessment of plasticizers in commercial samples.
\end{abstract}

Keywords: polyvinylchloride; plasticizers; vibrational techniques.

\section{INTRODUÇÃO}

O poli(cloreto de vinila) $\left(\mathrm{PVC},\left[\mathrm{CH}_{2}-\mathrm{CHCl}\right]_{\mathrm{n}}\right)$ é um dos polímeros mais usados atualmente, embora apresente, quando puro, elevada rigidez e sofra degradação quando exposto à luz solar ou mesmo a temperaturas perto da ambiente, com eliminação de cloreto de hidrogênio. ${ }^{1}$ A melhora na propriedade mecânica e na estabilidade do PVC é feita através da inserção de aditivos, tais como plastificantes e estabilizantes.

A adição de plastificantes diminui as forças intercadeias, aumentando a mobilidade das mesmas, tornando assim o polímero mais flexível e de fácil processamento. ${ }^{2}$ Os plastificantes podem ser classificados como internos ou externos. As moléculas de plastificante externo não estão ligadas à cadeia polimérica e podem ser liberadas por evaporação, extração ou difusão, enquanto que o plastificante interno é parte inerente da cadeia polimérica, oferecendo uma melhor estabilidade mecânica ao polímero. ${ }^{3}$ A adição de plastificantes ao PVC permite que este polímero seja vendido no comércio como filmes esticáveis utilizados como, por exemplo, em embalagens para alimentos em supermercados e na culinária doméstica. Os plastificantes externos do PVC mais usados na indústria são líquidos inodoros de coloração amarelada tênue, insolúveis em água e com alto ponto de ebulição. Dentre os plastificantes externos mais comuns estão o di(2-etil-hexil) ftalato e di(2-etil-hexil) adipato comumente abreviados e conhecidos pelas siglas em inglês DEHP e DEHA, cujas estruturas são apresentadas no Esquema 1.

Os ftalatos são utilizados há mais de cinqüenta anos como plastificantes do PVC em diversas finalidades. Entretanto, estudos toxicológicos recentes demonstraram que DEHP em ratos e camundongos causa embriotoxicidade, atrofia testicular e anomalias no sistema nervoso central. ${ }^{4}$ Em humanos a toxicidade destes plastificantes leva a problemas hepáticos. ${ }^{5,6}$ Nos Estados Unidos a US Consumer Product Safety Commission considerou o DEHP um produto carcinogênico ao ser humano. A via de exposição aos ftalatos do filme de PVC esticável deve-se principalmente à difusão dos plastificantes da matriz polimérica para os produtos alimentícios embalados, principalmente para alimentos que contenham uma alta porcentagem de gordura.

*e-mail: mlatempe@iq.usp.br

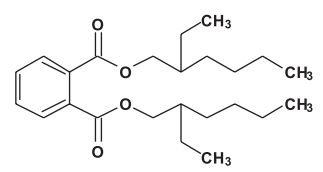

DEHP

di(2-etil-hexil) ftalato

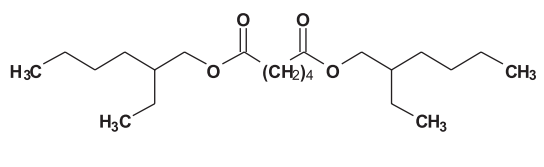

DEHA

di(2-etil-hexil) adipato

Esquema 1. Fórmulas estruturais de plastificantes para filmes de PVC esticável

Diversos órgãos governamentais e não governamentais no Brasil têm voltado suas atenções ao emprego do PVC em brinquedos, materiais hospitalares e em embalagens de alimentos. A legislação brasileira estabelece a permissão do uso de DEHP em quantidade não superior a $3 \%$ da matéria plástica para embalar alimentos contendo níveis superiores a 5\% de gordura. ${ }^{7}$ Tratando-se de um assunto de saúde pública, a restrição ao uso de plastificantes ftálicos em embalagens alimentícias tem sido abordada em diversos trabalhos, assim como a necessidade de sua determinação quantitativa e substituição por plastificantes menos nocivos à saúde humana. ${ }^{8}$

Atualmente algumas propostas de plastificantes alternativos aos ésteres ftálicos foram apresentadas. Alguns grupos sugerem a utilização de bioésteres do ácido cítrico como plastificantes, pois estes não possuem rejeição quando presentes em produtos alimentícios. ${ }^{9}$ Outros propõem como alternativa a utilização de líquidos iônicos. ${ }^{10}$ Tais plastificantes, porém apresentam preços elevados quando comparados aos 
ésteres de ftalatos normalmente utilizados. Atualmente, os adipatos são compostos economicamente viáveis e menos perigosos. ${ }^{11}$

Ao contrário do plastificante DEHP, não é observada qualquer disfunção renal ou toxicidade testicular resultante da administração de DEHA. Os resultados foram confirmados em ensaios em ratos ${ }^{12}$ e em seres humanos, observando-se que a administração de DEHA não aumenta a incidência ou disfunções como perda de peso, análise de esperma e histopatologia. ${ }^{13}$

A maioria dos filmes de PVC esticável utilizados em contato direto com alimentos, colocados à venda no comércio varejista brasileiro, não informam o tipo e a quantidade de plastificantes na composição declarada na embalagem.

As técnicas mais empregadas para análise qualitativa e quantitativa de plastificantes em diferentes matrizes poliméricas de PVC são cromatografia gasosa ${ }^{5}$ e cromatografia líquida de alta eficiência. ${ }^{14,15} \mathrm{Na}$ literatura são também encontrados alguns trabalhos onde a espectroscopia $\operatorname{Raman}^{11,16}$ e a espectroscopia no infravermelho ${ }^{17}$ são empregadas.

A identificação e análise de plastificantes no PVC têm sido estudadas por diversos grupos brasileiros de pesquisa. Morelli-Cardoso et al. ${ }^{14}$ quantificaram DEHP utilizando cromatografia líquida de alta eficiência em amostras simuladas de alimentos gordurosos e não gordurosos, que ficaram em contato com três diferentes marcas comerciais de filme de PVC esticável comprados em supermercados da cidade do Rio de Janeiro. Os autores concluíram que os filmes contemplavam as normas de qualidades vigentes, pois não constataram migração de DEHP para as amostras simuladas de alimentos. Freire et al. ${ }^{18}$ quantificaram por cromatografia gasosa os plastificantes de PVC utilizados nos filmes de PVC esticável e selo de fechamento de embalagem. Obtiveram para os filmes de PVC esticável entre 13 a $14 \%$ de DEHA e 20 a $22 \%$ de DEHP. Neste trabalho se alerta para a falta de informação da composição e restrição de uso nos rótulos das embalagens dos filmes de PVC esticável. Esteves et al. ${ }^{19}$ desenvolveram e avaliaram um método analítico para determinação de DEHA e DEHP em cerca de 50 filmes de PVC esticável disponíveis no mercado nacional. A técnica de cromatografia gasosa foi utilizada e foram avaliados diversos solventes para extração dos plastificantes. Os resultados indicaram que o clorofórmio apresentou o melhor desempenho tendo a maior taxa de recuperação ( $c a$. de 100\%) para um menor tempo de contato ( $c a$. de $1 \mathrm{~h}$ ). O hexano apresentou também bom desempenho, tendo sido utilizado no nosso estudo devido ao seu menor impacto ambiental em relação ao clorofórmio.

O objetivo do presente estudo foi aplicar as espectroscopias vibracionais de absorção no infravermelho e espalhamento Raman na identificação e quantificação dos plastificantes em alguns filmes comerciais de PVC esticável adquiridos em supermercados da cidade de São Paulo.

\section{PARTE EXPERIMENTAL}

\section{Reagentes e métodos de extração}

Os padrões de plastificantes DEHA e DEHP, líquidos levemente amarelados, foram fornecidos pela empresa Elekeiroz. Os solventes utilizados foram de grau analítico, tetra-hidrofurano (Synth), n-hexano (Aldrich), e poli(cloreto de vinila) sólido puro (Aldrich), utilizados sem qualquer tratamento prévio. Os filmes comerciais de PVC esticável de diferentes fabricantes e marcas comerciais, codificados de A a I, foram adquiridos em diferentes supermercados na cidade de São Paulo/SP.

A extração do plastificante para identificação qualitativa foi realizada utilizando dois solventes, através dos procedimentos descritos abaixo.

\section{Solvente polar (tetra-hidrofurano - THF $)^{18}$}

Em um béquer de vidro de $50 \mathrm{~mL}$ sob agitação à temperatura ambiente, são adicionados $20 \mathrm{~mL}$ de THF em aproximadamente $0,5 \mathrm{~g}$ do filme de PVC esticável. Após a solubilização total do filme é cessada a agitação. Com o auxílio de uma pipeta de Pasteur são adicionados $25 \mathrm{~mL}$ de etanol à solução sem agitação, ocorrendo a formação do PVC sólido, branco e pouco denso. A separação das duas fases é realizada através de filtração simples, e o filtrado é rotoevaporado obtendo-se um líquido pouco viscoso, amarelo claro.

\section{Solvente apolar (n-hexano)}

Em um béquer de $50 \mathrm{~mL}$ à temperatura ambiente contendo aproximadamente $1 \mathrm{~g}$ de filme comercial de PVC esticável finamente cortado, foram adicionados $40 \mathrm{~mL}$ de $\mathrm{n}$-hexano. $\mathrm{O}$ sistema foi mantido fechado em repouso por $2 \mathrm{~h}$. Após este tempo a solução foi retirada do béquer. Verificou-se que o PVC apresentou uma diminuição da flexibilidade como consequência da difusão do plastificante para o solvente apolar. Ao béquer ainda contendo o filme comercial de PVC menos flexível, foi adicionada uma nova alíquota de $25 \mathrm{~mL}$ de n-hexano e mantido fechado sob repouso durante $3 \mathrm{~h}$. Este último processo foi repetido mais uma vez. Os três extratos foram rotoevaporados até restar um líquido viscoso amarelo claro.

\section{Técnicas espectroscópicas}

\section{FT-Raman}

Os espectros FT-Raman foram obtidos no interferômetro da Bruker, RFS 100 com detector de Ge refrigerado com nitrogênio líquido e radiação excitante em $1064 \mathrm{~nm}$ (laser Nd:YAG). A potência do laser foi mantida em $100 \mathrm{~mW}$ e para cada espectro foram feitas 256 varreduras utilizando-se resolução de $4 \mathrm{~cm}^{-1}$. Para as amostras em solução foi utilizada uma cubeta de quartzo, que é fixada a um porta-amostra contendo um lado espelhado para reflexão interna do laser na cubeta. As amostras em filme foram fixadas diretamente em um porta-amostra.

\section{FT-IR}

Os espectros FT-IR foram obtidos no interferômetro da Bomem MB-100. Para cada espectro foram efetuadas 32 varreduras utilizandose resolução de $4 \mathrm{~cm}^{-1}$. Os plastificantes extraídos (líquido viscoso) foram colocados entre placas de $\mathrm{KBr}$ e os filmes comerciais foram presos por molas à janela de um porta-amostra adequado.

\section{Procedimento para quantificação dos plastificantes por FT-IR}

\section{Análise quantitativa através da espectroscopia IR}

As análises quantitativas foram realizadas através do método de adição de padrão, o qual tem por objetivo eliminar as interferências de espécies não conhecidas presentes junto com o analito (efeito da matriz). Nesse método, a curva padrão é construída adicionando-se alíquotas do padrão (plastificante) a uma matriz formada pelo PVC puro e o filme de PVC esticável comercial, que será analisado. O mérito deste método é que as determinações são realizadas em um meio de análise muito semelhante, sendo a concentração do plastificante padrão adicionado o diferencial entre as amostras.

A curva padrão para a análise quantitativa para cada filme foi preparada utilizando béqueres de vidro de $50 \mathrm{~mL}$, onde foram colocados aproximadamente $0,05 \mathrm{~g}$ do analito (filme comercial de PVC esticável) e 0,04 g do PVC sólido puro que foram dissolvidos em ca. $4 \mathrm{~mL}$ de THF. Após a dissolução completa foram adicionadas massas de plastificante padrão correspondentes a 5, 10, 15, 20, 25 e $30 \% \mathrm{~m} / \mathrm{m}$. 
Os filmes dessas soluções foram formados por completa evaporação do solvente, em lâminas de microscópio de dimensões $3 \times 3 \mathrm{~cm}$, previamente limpas, que foram imersas nas soluções de THF contida nos béqueres. Os espectros IR foram obtidos dos filmes retirados das lâminas de vidro. Foram medidas as áreas da banda em $c a .1730$ $\mathrm{cm}^{-1}$, atribuída ao estiramento da carbonila dos plastificantes, ${ }^{20} \mathrm{e}$ da banda em $1251 \mathrm{~cm}^{-1}$ (deformação angular $\left.\mathrm{Cl}-\mathrm{C}-\mathrm{H}\right)^{21}$ do $\mathrm{PVC}$ puro. Essas bandas foram escolhidas por serem bandas de intensidade forte e média, e por serem bem separadas das demais bandas. As áreas das bandas foram calculadas utilizando o método de integração do programa Grams (GRAMS/32 software - Galactic Industries Company, Salem, NH/EUA). O desvio na medida da área da banda foi de aproximadamente $0,6 \%$.

O desvio da porcentagem de plastificantes totais foi considerado igual ao desvio da equação da reta experimental.

\section{RESULTADOS E DISCUSSÃO}

\section{Análise qualitativa}

Os espectros Raman do polímero de PVC puro em pó dos padrões DEHP e DEHA líquidos e do filme de PVC esticável codificado como "A" são apresentados na Figura 1. É possível observar que o filme de PVC esticável e os padrões de plastificantes possuem em comum a banda em aproximadamente $1730 \mathrm{~cm}^{-1}$ atribuída ao estiramento do grupo carbonílico. Esta banda está ausente no espectro do PVC puro. Em todos os espectros são observadas bandas em aproximadamente 1450 e $2900 \mathrm{~cm}^{-1}$, atribuídas a vibrações angulares (H-C-H) no plano e aos estiramentos $(\mathrm{C}-\mathrm{H})$ dos grupos alquilas presentes em todos os materiais analisados. ${ }^{11}$

No plastificante DEHP, por ter em sua estrutura um anel aromático, são observadas em seu espectro Raman as bandas em 653, 1041 e 1580-1600 $\mathrm{cm}^{-1}$ correspondentes a vibrações de anel aromático orto-dissubstituído, ${ }^{16}$ e a banda em $3071 \mathrm{~cm}^{-1}$ atribuída ao estiramento carbono-hidrogênio de anéis aromáticos. ${ }^{20}$ Essas bandas estão ausentes nos espectros do padrão DEHA e do PVC puro, portanto, podem ser utilizadas como bandas características do DEHP. Para o plastificante DEHA não foram encontradas bandas que pudessem ser observadas unicamente no espectro Raman deste plastificante. No espectro do filme comercial de marca A não foram observadas as bandas do DEHP, sugerindo que o plastificante em maior quantidade seja o DEHA. São também observadas as bandas em 637, 694 e 1430 $\mathrm{cm}^{-1}$ do PVC puro.

Na Figura 2 são apresentados os espectros Raman dos plastificantes extraídos dos filmes comerciais e dos padrões DEHA e DEHP. Não foram observadas diferenças entre os espectros Raman dos plastificantes obtidos por extração em THF e em n-hexano. A observação da banda em $3071 \mathrm{~cm}^{-1}$, juntamente com a das bandas em 653,1041 e $1580-1600 \mathrm{~cm}^{-1}$ indicam a presença de DEHP no filme de marca I. Já os espectros dos plastificantes extraídos dos filmes de marcas A, B, C, E e H apresentam a banda em $3071 \mathrm{~cm}^{-1}$, com fraca intensidade relativa, o mesmo ocorrendo para as bandas devido ao anel aromático em 653, 1041 e $1580-1600 \mathrm{~cm}^{-1}$. A intensidade relativa da banda em $1450 \mathrm{~cm}^{-1}$ e do estiramento carbonílico nesses espectros é semelhante àquela observada no espectro do DEHA. Essas observações indicam que esses filmes contêm DEHP em menor quantidade que o DEHA. Os espectros dos plastificantes extraídos dos filmes de marca D, F e G não apresentam a banda em $3071 \mathrm{~cm}^{-1}$, sugerindo a ausência DEHP em sua composição ou uma menor quantidade do mesmo nestas marcas em relação às demais analisadas. As intensidades relativas das bandas nesses espectros são semelhantes às do DEHA, indicando a predominância deste plastificante nesses filmes.

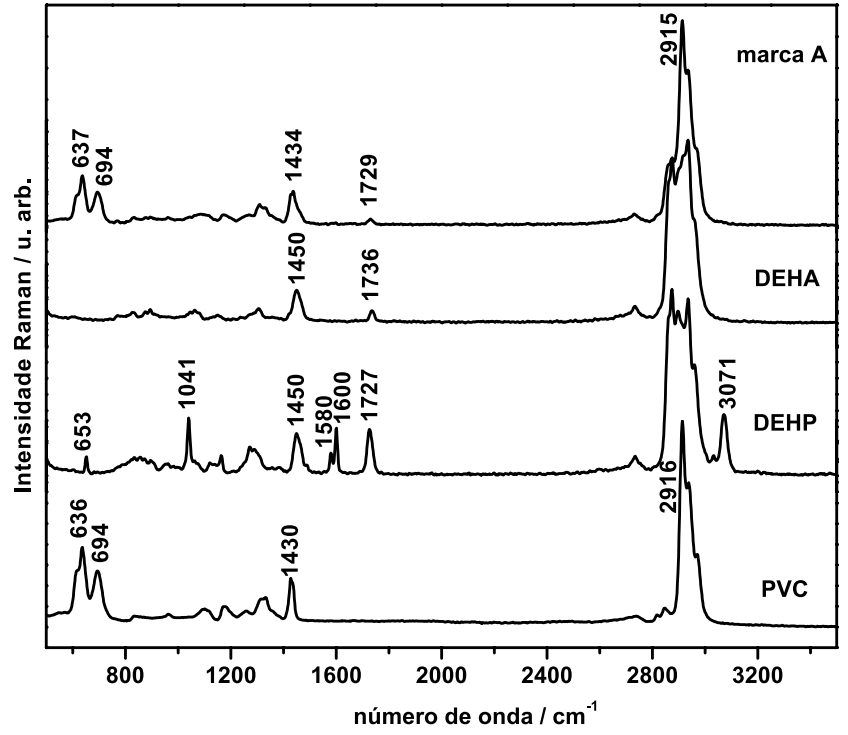

Figura 1. Espectros FT-Raman do filme de PVC esticável da marca A, dos líquidos plastificantes padrão DEHA e DEHP e do polímero (PVC) puro em pó

Se compararmos o espectro do filme comercial codificado como A na Figura 1 com o espectro do plastificante extraído do filme A na Figura 2, verificamos que a banda em $3071 \mathrm{~cm}^{-1}$ não é observada no espectro da Figura 1 sendo, porém, observada com fraca intensidade no espectro da Figura 2 (indicada com um asterisco). Este comportamento se deve à baixa concentração do plastificante DEHP no filme comercial A. A extração dos plastificantes aumenta a quantidade relativa de DEHP na amostra tornando possível a observação da banda em $3071 \mathrm{~cm}^{-1}$ no espectro desses plastificantes. Na Figura 2 são mostrados os espectros dos plastificantes extraídos dos filmes ao invés dos espectros Raman dos filmes comerciais.

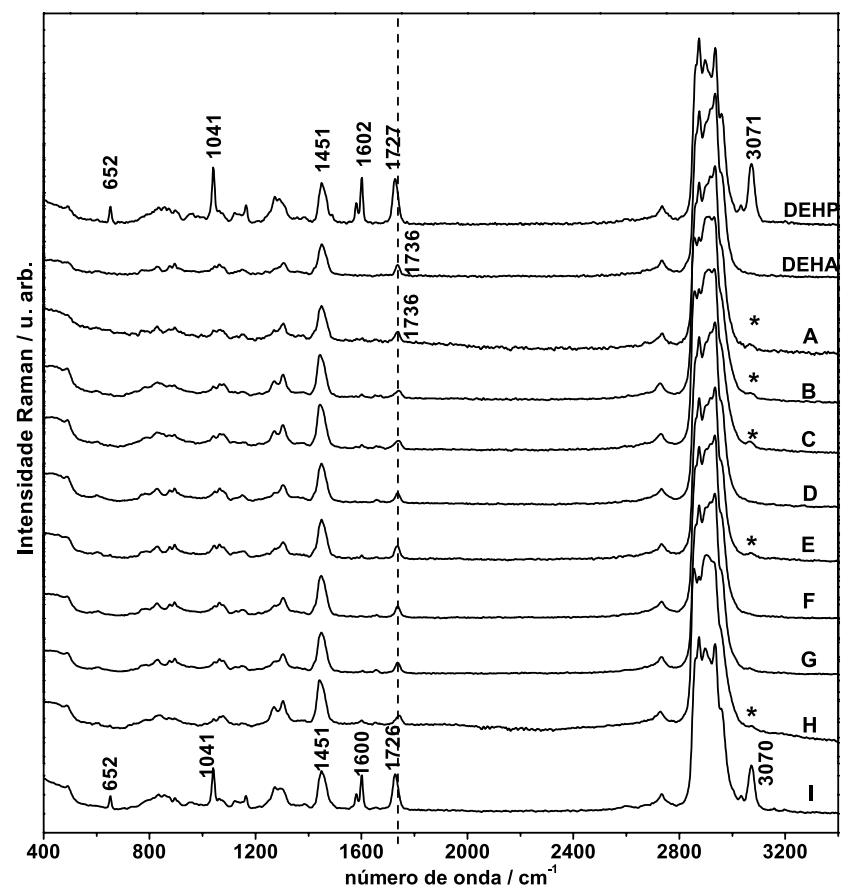

Figura 2. Espectros Raman dos líquidos plastificantes padrão DEHP e DEHA, e dos plastificantes extraídos das diferentes marcas de A a I 
Na Figura 3 são apresentados os espectros IR dos plastificantes padrões e os dos plastificantes extraídos dos filmes de PVC esticável comerciais. Observa-se que o estiramento da carbonila em $c a .1730 \mathrm{~cm}^{-1}$ é a banda mais intensa dos espectros dos plastificantes. Comparando os espectros do DEHP e do DEHA, as bandas finas e de altas intensidades relativas em $743 \mathrm{~cm}^{-1}$ (deformação do $\mathrm{C}-\mathrm{H}$ fora do plano do anel), 1073 $\mathrm{cm}^{-1}$ (deformação do C-H ou C-C no plano do anel), 1124 e $1277 \mathrm{~cm}^{-1}$ (deformação do C-H no plano do anel) e as bandas finas e de fracas intensidades em 1580 e $1600 \mathrm{~cm}^{-1}$ (estiramento $\mathrm{C}-\mathrm{C}$ do anel) ${ }^{22}$ podem ser utilizadas como bandas características do DEHP. O espectro FT-IR do DEHA apresenta em $1170 \mathrm{~cm}^{-1}$ uma banda larga com três componentes, sendo a mais intensa a central em $1175 \mathrm{~cm}^{-1}$. Este formato de banda é observado na maioria dos espectros dos plastificantes extraídos, com exceção do espectro dos plastificantes do filme I. Neste espectro são observadas somente as bandas correspondentes ao padrão DEHP. A banda em $1277 \mathrm{~cm}^{-1}$, devida ao DEHP, não é observada nos espectros dos plastificantes extraídos dos filmes $\mathrm{D}, \mathrm{Fe} \mathrm{G}$ indicando ausência ou pouca quantidade do DEHP nesses filmes. Esses resultados são concordantes com aqueles obtidos por espectroscopia Raman.

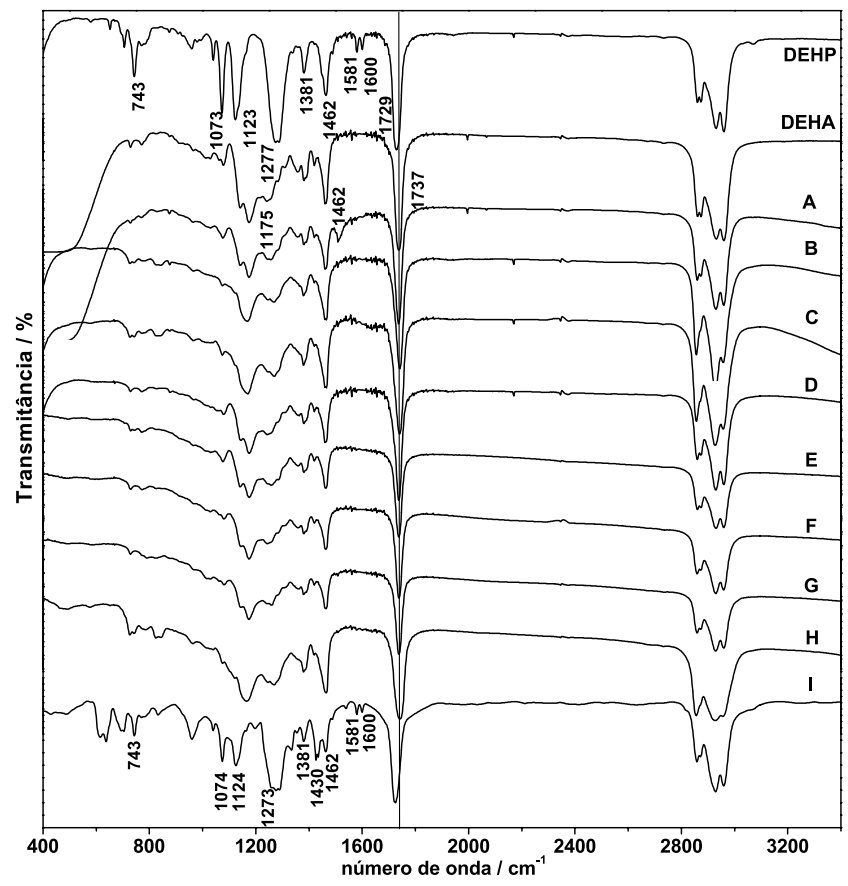

Figura 3. Espectros FT-IR dos líquidos plastificantes padrão DEHA, DEHP e dos plastificantes extraídos das marcas de A a I

É interessante comparar a região de $3050 \mathrm{~cm}^{-1}$ no espectro FTRaman (Figura 2) e no infravermelho dos plastificantes extraídos do filme comercial I, predominantemente DEHP. Vemos a ausência no espectro no infravermelho da banda em $3071 \mathrm{~cm}^{-1}$, devida ao estiramento C-H do anel benzênico observada no espectro Raman. Isto se deve à diferença na natureza física das duas técnicas (processo absorção no infravermelho e espalhamento no Raman). No infravermelho a intensidade de uma banda é tanto maior quanto maior for a variação do momento de dipolo da molécula durante a vibração, como essa variação é pequena durante o estiramento $\mathrm{C}-\mathrm{H}$ do anel benzênico esta banda não é observada no espectro infravermelho do filme I.

\section{Análise quantitativa}

Na determinação quantitativa dos plastificantes por espectroscopia no IR, a espessura do filme é um fator que contribui para a inten- sidade da banda de absorção medida, além da absortividade molar e da concentração dos plastificantes. Para eliminar a necessidade de controle das espessuras dos filmes foram tomadas as intensidades relativas das bandas (áreas das bandas) em $c a .1730 \mathrm{~cm}^{-1}$, atribuída ao estiramento da carbonila, e a banda em $1251 \mathrm{~cm}^{-1}$ do PVC puro, atribuída à deformação angular $\mathrm{Cl}-\mathrm{C}-\mathrm{H},{ }^{21} \mathrm{e}$ as absortividades molares das espécies foram consideradas iguais. Devido à pequena diferença de frequência entre as bandas da carbonila do DEHP e DEHA não é possível medir, de maneira separada, a intensidade de cada componente na banda. Obtém-se, portanto, por IR, uma medida que se refere à porcentagem total dos dois plastificantes.

Na Figura 4 são apresentados, como exemplo, os espectros IR do filme de PVC esticável marca D, do plastificante extraído deste filme e do PVC puro. Também estão presentes os gráficos $I_{\text {plast. }}=\mathrm{A}_{1738 \mathrm{~cm}(-1)} / \mathrm{A}_{1251 \mathrm{~cm}(-1)}$ (razão das áreas das bandas indicadas) em função da porcentagem em massa de DEHA adicionado em cada amostra contendo PVC puro e filme comercial. Esses gráficos foram utilizados na determinação dos plastificantes através do método de adição de padrão. Foram escolhidas cinco determinações que refletem a qualidade dos resultados obtidos, com seus respectivos coeficientes de correlação. O desvio-padrão para cada ponto apresentado no gráfico foi obtido a partir de um filme seccionado em três partes.

Como pode ser notado na Figura 4, o método para os filmes B e D apresenta boa linearidade (coeficiente de correlação $=0,9999$ e 0,9978), os filmes A e I apresentam linearidade média (coeficiente de correlação $=0,9921$ e 0,996) e o filme E apresenta uma linearidade regular (coeficiente de correlação $=0,961$ ). A análise pela curva padrão para o filme de marca A apresenta $17,4 \%$, marca B apresenta $11,9 \%$, marca D apresenta $16,7 \%$, marca E apresenta $22 \%$ e marca I apresenta $19,9 \%$ de plastificantes totais, e estes valores se aproximam dos valores indicados pelas literaturas consultadas. ${ }^{18}$

Utilizando a curva padrão pela técnica de infravermelho, foram encontrados teores de plastificantes totais nos filmes comerciais de PVC esticável avaliados que variam entre 12 e 27\%. Na Tabela 1 são apresentados os resultados obtidos por IR para os filmes comerciais de PVC esticável.

Tabela 1. Concentrações percentuais dos plastificantes totais extraídos dos filmes comerciais de PVC esticável de marcas A - I

IR

Filme $\quad$ Plastificantes totais (\%)

\begin{tabular}{lc}
\hline A & $17,4 \pm 0,7$ \\
B & $11,9 \pm 0,1$ \\
C & $27,4 \pm 0,8$ \\
D & $16,7 \pm 0,2$ \\
E & $22 \pm 1$ \\
F & $17 \pm 2$ \\
G & $19,4 \pm 0,5$ \\
H & $18 \pm 2$ \\
I & $19,9 \pm 0,6$ \\
\hline
\end{tabular}

Interessante notar que os espectros por FT-Raman e FT-IR indicam o DEHP como plastificante majoritário do filme comercial codificado por I. O uso deste plastificante é vetado pela legislação brasileira. Também para os filmes comerciais D, F e G tanto os resultados por FT-Raman como por FT-IR indicam que o plastificante presente é essencialmente DEHA. 


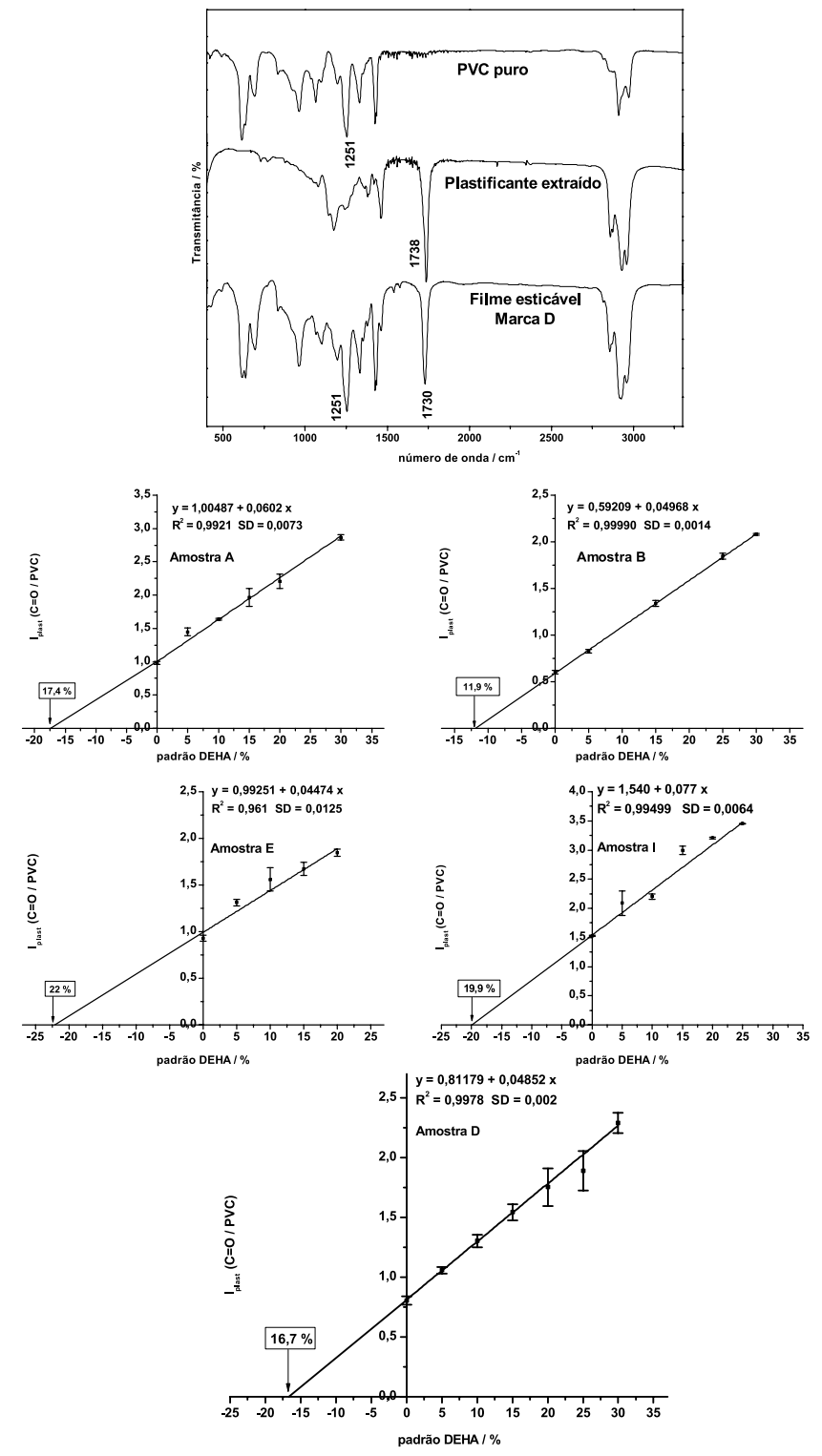

Figura 4. Espectros FT-IR do PVC puro, do plastificante extraído e do filme de PVC esticável da marca D e curvas padrão obtidas por FT-IR para os filmes de marcas $A, B$, E, I e $D$

\section{CONCLUSÃO}

Este trabalho mostrou a aplicação das espectroscopias Raman e FT-IR para identificação e quantificação dos plastificantes no filme de PVC esticável comercial. Considerando a praticidade e o baixo custo da análise utilizando a técnica FT-IR, os resultados obtidos indicam que esta técnica possa ser usada para uma primeira avaliação dos plastificantes existentes em filmes comerciais de PVC esticável.

\section{AGRADECIMENTOS}

Ao apoio financeiro da FAPESP. M. L. Souza agradece as bolsas recebidas da FAPESP e CNPq. M. L. A. Temperini e P. Corio agradecem ao CNPq pelas bolsas de produtividade em pesquisa. Os autores agradecem ao técnico M. N. Wandermuren, da Central Analítica do IQ-USP e à Profa. M. F. M. Tavares pelas frutíferas discussões.

\section{REFERÊNCIAS}

1. Vinhas, G. M.; Souto-Maior, R. M.; Almeida, Y. M. B.; Polym. Degrad. Stab. 2004, 83, 429.

2. Crespo, J. E.; Balart, R.; Sanches, L.; Lopez, J.; J. Appl. Polym. Sci. 2007, 104, 1215.

3. Rahman, M.; Brazel, C. S.; Prog. Polym. Sci. 2004, 29, 1223; Vinhas, G. M.; Souto-Maior, R. M.; Almeida, Y. M. B.; Polímeros: Ciência e Tecnologia 2005, 15, 207.

4. Heudorf, U.; Mersh-Sudermann, V.; Angerer, J.; Int. J. Hyg. Environ. Health 2007, 210, 623.

5. Marin, M. L.; López, J.; Sánchez, A.; Vilaplana, J.; Jiménez, A.; Bull. Environ. Cont. Toxicol. 1998, 60, 68.

6. Lattini, G.; Felice, C.; Verrotti, A.; Reprod. Toxicol. 2004, 19, 27.

7. http://e-legis.anvisa.gov.br/leisref/public/home.php, Resolução $\mathrm{n}^{\circ} 105$, de 19 de maio de 1999, acessada em Fevereiro 2008.

8. Atek, D.; Belhaneche-Bensemra, N.; Eur. Polym. J. 2005, 41, 707; Petersen, J. H.; Togestov, P.; Hallas, J.; Olsen, M. B.; J rgensen, B.; Jakobsen, M.; Packag. Technol. Sci. 2004, 17, 53.

9. Gil, N.; Saska, M.; Negulesco, I.; J. Appl. Polym. Sci. 2006, 102, 1366.

10. Rahman, M.; Brazel, C. S.; Polym. Degrad. Stab. 2006, $91,3371$.

11. Berg, R. W.; Otero, A. D.; Vib. Spectrosc. 2006, 42, 222.

12. Nabae, K.; Dói, Y.; Takahashi, S.; Ichihara, T.; Toda, C.; Ueda, K.; Okamoto, Y.; Kojima, N.; Tamano, S.; Shirai, T.; Reprodutive Toxicology 2006, 22, 411; Kang, J. S.; Morimura, K.; Toda, C.; Wanibuchi, H.; Wei M.; Kojima N.; Fukushima S.; Reprodutive Toxicology 2006, 21, 253.

13. Jarfelt K.; Dalgaard, M.; Hass, U.; Borch, J.; Hacobsen, H.; Ladefoged, O.; Reprodutive Toxicology 2005, 19, 505.

14 Morelli-Cardoso, M. H. W.; Lachter, E. R.; Tabak, D.; Abrantes, S.; de Moraes, O. M. G.; J. High Resol. Chromatog. 1999, 22, 70.

15. Aignasse, M. F.; Prognon, P.; Stachowicz, M.; Gheyouche, R.; Pradeaus, D.; Int. J. Pharmaceutics 1995, 113, 241.

16. Nfrbygaard, T.; Berg, R. W.; Appl. Spectrosc. 2004, 58, 410.

17. Chan, W. H.; J. Chem. Educ. 1987, 64, 897.

18. Freire, M. T. A.; Santana, I. A.; Reyes, F. G. R.; Food Addit. Contam, 2006, 23, 93.

19. Esteves, A. A.; Borges, S. V.; Quim. Nova 2007, 30, 219

20. Reyes-Labarta, J.; Herrero, M.; Tiemblo, P.; Mijangos, C.; Reinecke, H.; Polymer 2003, 44, 2263; Nyquist, R. A.; Appl. Spectrosc. 1972, 26, 81.

21.Painter, P. C.; Coleman, M. M.; Koenig, J. L.; The Theory of Vibrational Spectroscopy and its Application to Polymeric Materials, Wiley-Interscience: New York, 1982, p. 406.

22. Loring, J. S.; Karlsson, M.; Fawcett, W. R.; Casey, W. H.; Spectrochim. Acta, Part A 2001, 57, 1635; Arenas, J. F.; Marcos, J. I.; Spectrochim. Acta, Part A 1979, 35, 355; Yan, C.; Zhang, Y.; Hu, Y.; Ozaki, Y.; Shen, D.; Gan, Z.; Yan, S.; Takahashi, I.; J. Phys. Chem. B 2008, 112, 3311. 